\title{
Intussusception in the adult: clinical, radiological and histological features
}

\author{
Stephen P. Courtney, Nassif Ibrahim ${ }^{1}$, Andrew J. Longstaff ${ }^{2}$ \\ and Colin M. Davidson
}

Departments of General Surgery, ${ }^{1}$ Pathology and ${ }^{2}$ Radiology, Frenchay Hospital, Bristol, BSI6 ILE, UK

\begin{abstract}
Summary: Intussusception in the adult is an unusual cause of bowel obstruction. Unlike in childhood the clinical presentation is not clear cut and there are no distinct pathognomonic features. The radiological features are variable. Five patients presented to Frenchay Hospital over a five month period. The patients' clinical courses demonstrate the differing presenting features. Ultrasound investigation and CT scanning may show characteristic signs. Surgical treatment is mandatory as there is nearly always an underlying pathological abnormality which may be malignant.
\end{abstract}

\section{Introduction}

Intussusception is a process whereby one part of the gut becomes invaginated into an immediately adjacent segment. It is the commonest cause of intestinal obstruction in children. ${ }^{1}$ It is rarely encountered in adults accounting for between two and three cases in a population of $1,000,000$ per annum. ${ }^{2}$ In children the cause of intussusception is said to be idiopathic in $90 \%$ of cases. In nearly half the infants there is evidence of adenovirus infection, producing enlargement of Peyer's patches, which act as the lead point of the intussusception. ${ }^{3,4}$ Treatment is usually non-operative by barium hydrostatic reduction. By contrast, intussusception in the adult is rarely idiopathic and the treatment is surgical resection. The diagnosis is often made only at laparotomy ${ }^{5}$ and most surgeons have limited experience of this condition and its diagnosis.

Five adults with intussusception presented in a five-month period to Frenchay Hospital serving a population of 220,000 people. They illustrate the varying clinical presentations, radiological modalities used to diagnose intussusception and differing pathological basis.

\section{Patients}

\section{Patient 1}

A 69 year old man presented with a history of two days colicky abdominal pain which had occurred

Correspondence: S.P. Courtney, M.Ch., F.R.C.S., Ysbyty Gwynedd, Bangor, Gwynedd LL57 2PW, UK. Accepted: 10 December 1991 two weeks prior to his clinic appointment. He had associated loose bowel motions and passed blood per rectum. His abdomen had not been distended.

On examination there was a tender palpable caecum. Full blood count, electrolytes and liver function tests were normal. An ultrasound scan performed two days prior to attending clinic dido not show a caecal mass. A barium enema showed a? small polyp in the mid-transverse colon and a defect on the medial wall of the caecum.

Following barium enema examination he was seen in clinic and admitted because of loss of appetite, vomiting and constipation. A tender mass was palpable in the right iliac fossa. A computed tomographic (CT) scan showed a mass in the ascending colon with a gas-filled centre surrounded by concentric layers, presumed to be bowel wall, free fluid and a surrounding abscess cavity wall (Figure 1). At laparatomy a large mass involving terminal ileum, caecum and ascending colon was resected. An ileo-colic intussusception was found with a warty lesion measuring $5 \times 4 \mathrm{~cm}$ on the caecum forming the tip of the intussusceptum. Histology showed a well-differentiated, Duke's A, adenocarcinoma of caecum. The intussuscepted caecum presumably accounted for the defect seen on barium enema.

\section{Patient 2}

A 67 year old man presented as an emergency with colicky abdominal pain and absolute constipation for 24 hours. On examination he had a distended, tympanitic, non-tender abdomen. Plain abdominal radiographs confirmed a small bowel obstruction with the typical features of dilated small bowel 


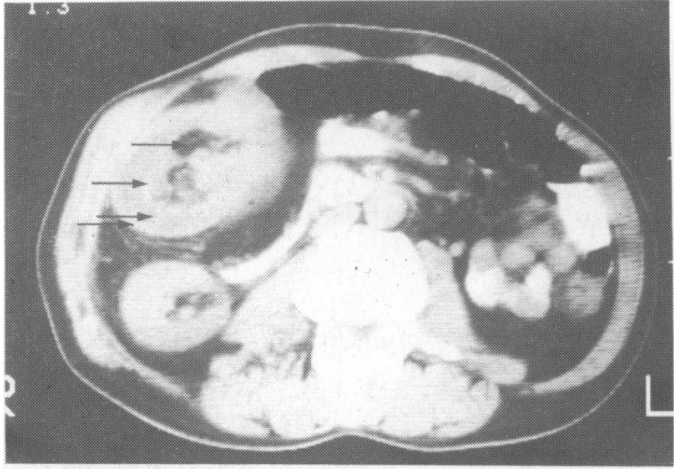

Figure 1 CT scan of right iliac fossa mass showing concentric rings (arrowed) in the top left corner of the picture (patient 1).

loops and multiple fluid levels. He was managed with intravenous fluids and nasogastric aspiration but two hours later was noted to be tender in the right iliac fossa.

At laparotomy he was found to have an ileo-ileal intussusception which spontaneously reduced. The section of bowel involved in the intussusception was resected. Histology showed an infiltrating angiolipoma with ulceration on the mucosal surface at the apex of the intussusception.

\section{Patient 3}

A 75 year old man was admitted as an emergency with a three week history of colicky central abdominal pain which had become much more severe on the day of admission. He had been constipated for 48 hours. On examination a nontender mass was palpable in the right iliac fossa beneath an appendicectomy scar. A preliminary diagnosis of adhesive obstruction was made and the patient treated with intravenous fluids and nasogastric aspiration. Ultrasound of the patient showed a complex mass illustrating the concentric ring and hayfork sign consistent with intussusception (Figures 2a, b and 3). At laparotomy an ileo-ileal intussusception with infarcted bowel was resected, at the tip of which was a gangrenous polypoid lesion measuring $4 \times 3 \times 2 \mathrm{~cm}$. Histology showed a benign submucosal angiolipoma consisting of mature adipose tissue intermingled with numerous blood vessels.

\section{Patient 4}

A 58 year old man presented with a long history of recurrent anaemia, epigastric pain and indigestion. On more than one occasion positive faecal occult blood had been detected but gastroscopy, barium meal and barium enema had been normal. Various a

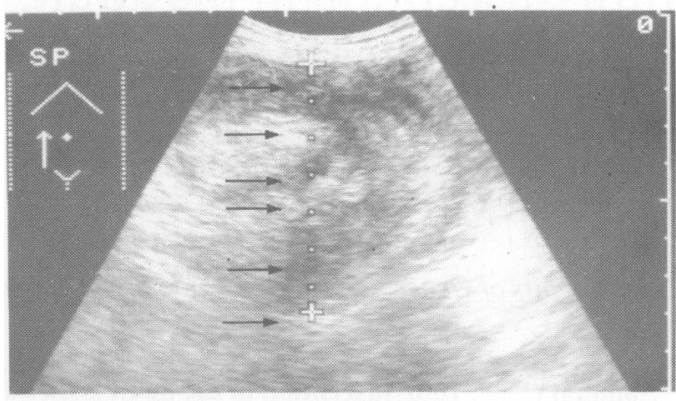

b

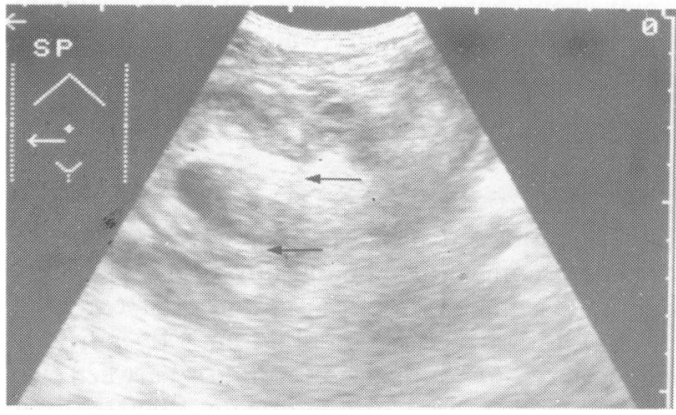

Figure 2 (a) Ultrasound scan of abdominal mass showing the concentric ring sign (arrowed) (patient 3). (b) Ultrasound scan of abdominal mass showing hayfork sign. The two limbs of hayfork arrowed (patient 3 ).

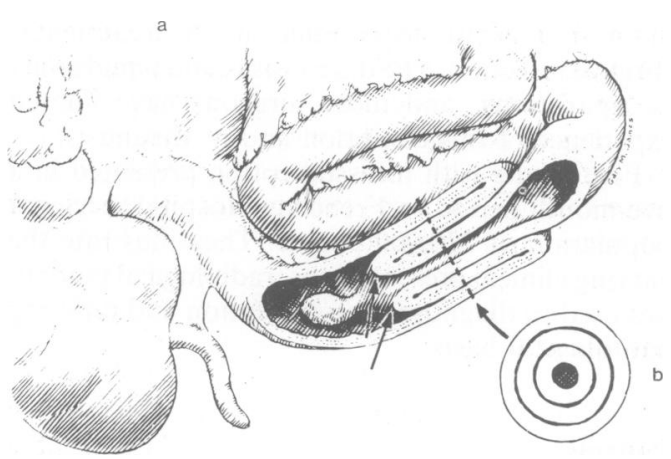

Figure 3 Diagrammatic illustration of (a) 'hayfork' sign (limbs of hayfork arrowed), and (b) concentric ring sign on ultrasound scan and CT scan.

empirical treatments with $\mathrm{H}_{2}$ antagonists and iron supplements had been tried without relief of symptoms.

A small bowel enema was arranged which demonstrated an intraluminal filling defect, possibly an 
inverted Meckel's diverticulum (Figure 4a).

At operation an ileo-ileal intussusception was noted which on manual reduction revealed a pit in the side wall.

The resected small segment of ileum showed a small dimple on the ante-mesenteric border with roughened serosa and a blood vessel disappearing within the dimple. Extending from the latter to the inside of the bowel was an inverted sausage-shaped thick-walled diverticulum measuring $5.5 \mathrm{~cm}$ in length and $1.3 \mathrm{~cm}$ in diameter (Figure $4 \mathrm{~b}$ ). Histology confirmed the diagnosis of Meckel's diverticulum lined partly by small bowel mucosa and partly by gastric mucosa. At the tip of the diverticulum there was a benign chronic peptic ulcer surrounded by gastric-type mucosa. It appears likely that this man's positive faecal occult blood, epigastric pain and anaemia were due to the presence of this peptic ulcer.

\section{Patient 5}

A 66 year old man was referred with a history of upper abdominal pain and gall stones on ultrasound scan. Gastroscopy was normal. Previously a malignant melanoma had been excised from his back with a right axillary block dissection. He underwent cholecystectomy and at laparotomy no other untoward features were noted. He made an uncomplicated recovery but when reviewed in clinic the abdominal pain had recurred being mainly left upper quadrant radiating into the back. There was associated borborygmi and then relief with episodes lasting $1 \frac{1}{2}$ hours for each of the two preceding days. He was immediately admitted to the ward and a small bowel enema arranged which showed an incomplete obstruction of the jejunum. At laparotomy a jejuno-ileal intussusception was noted. A small bowel resection was carried out.

The intussusception contained two polypoid masses measuring approximately $2.5 \mathrm{~cm}$ in diameter which were metastatic malignant melanoma on histological examination.

\section{Discussion}

In the United Kingdom intussusception in adults is a rare cause of bowel obstruction with a variable presenting clinical picture. In our patients, these varied from a long history over many years for the patient with Meckel's diverticulum (patient 4) (although arguably his intussusception was a later event) to a history over a few weeks with and without signs of obstruction (patients 1 and 3) and with the presence of a right iliac fossa mass; to acute small bowel obstruction (patients 2 and 5).

The radiological modalities used in an attempt to diagnose the small bowel obstruction also varied.

\section{a}
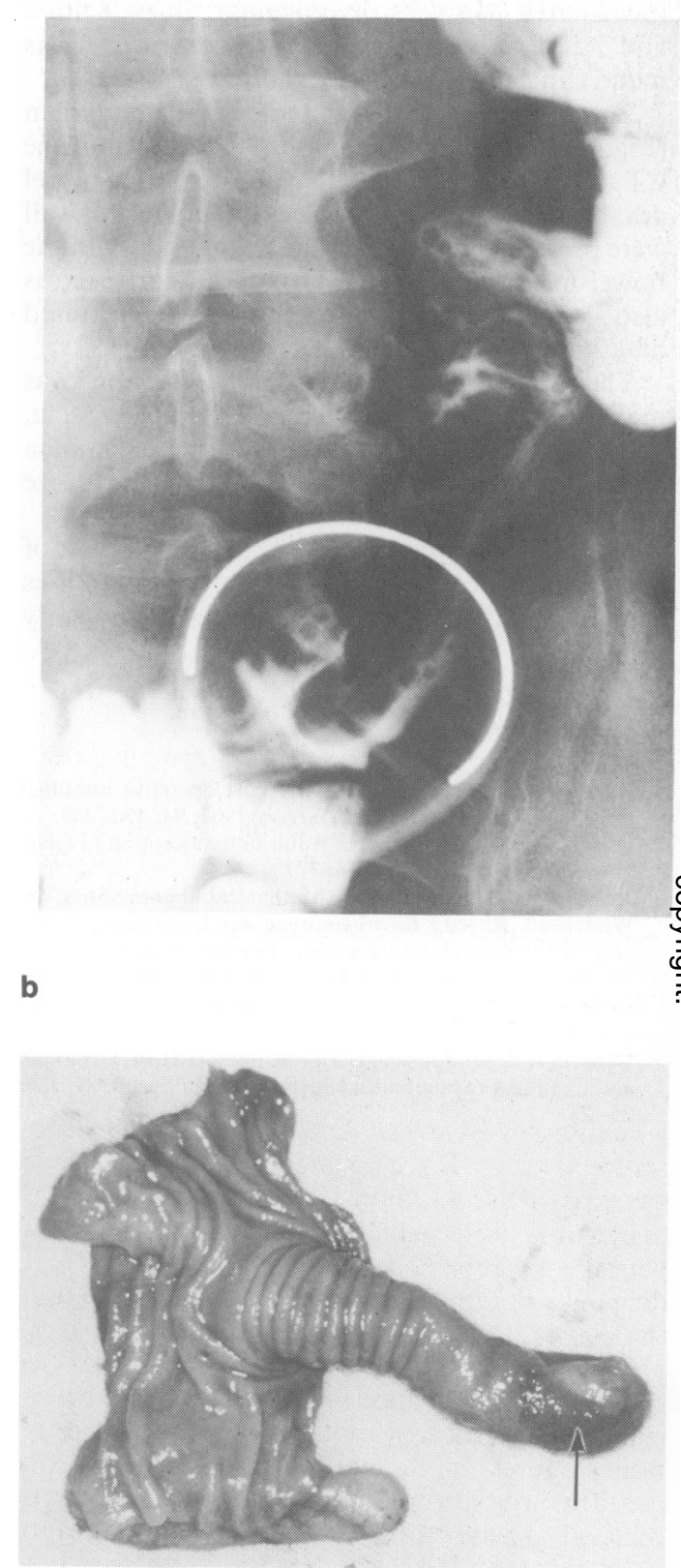

Figure 4 (a) Small bowel enema demonstrating an intraluminal filling defect consistent with an invaginated Meckel's diverticulum (seen within white ring) (patient 4). (b) Opened specimen showing inverted Meckel's diverticulum (gastric mucosa containing ulcer arrowed) (patient 4).

The diagnosis of intussusception was made preoperatively in two patients. The concentric ring and hayfork sign on ultrasound scan in patient 3 and in 
patient 4 the small bowel enema demonstrated the invaginated Meckel's diverticulum. In patients 2 and 5 the diagnosis of small bowel obstruction was made clinically but not the cause.

The concentric ring sign on ultrasound scan (Figure 2a) previously described ${ }^{6,7}$ was seen in the CT scan of patient 1 . The concentric rings of bowel gas, bowel wall, intraluminal fluid and bowel wall were present and merge on the medial side with the bowel mesentery (Figure 1). The hayfork sign is also seen in patient 3 . The features are illustrated diagrammatically in Figure 3.

The large bowel lesion of the caecum was malignant and the small bowel lesions benign, apart from the metastatic malignant melanoma which has a well-known predilection to metastasize to this site and cause intussusception. ${ }^{8,9}$

Operative intervention is indicated in all cases of adult intussusception. The risk of infarction is significant. As there is a causative lesion in nearly all cases resection is mandatory, even when reduction is easy with no evidence of bowel infarction or underlying pathology. In the previous 7 years at Frenchay Hospital only five other cases of intussusception in adults were recorded each with causative pathology. This is the experience of most authors except those in Glasgow. ${ }^{2}$ Bowel resection should be carried out in all cases as intraluminal lesions post reduction may be difficult to palpate, as are mural lesions such as infiltrating lipomata. Failing to undertake resection of an intussusception in an adult will always beg the question of whether a causative lesion, which may be malignant, was missed.

\section{Acknowledgements}

We thank the Department of Medical Illustration, Bristol Royal Infirmary and Frenchay Hospital for producing the figures.

\section{References}

1. Raffensperger, J.G. \& Baker, J.R. Post operative intestinal obstruction in children. Arch Surgery 1967, 94: 450-459.

2. Carter, C.R. \& Morton, A.L. Adult intussusception in Glasgow, UK. Br J Surg 1989, 76: 727.

3. Jones, P.F. \& Williams, R.A. Mechanical abnormalities. In: Whitehead, R. (ed.) Gastrointestinal and Oesophageal Pathology 1989, Churchill Livingstone, London, p. 319.

4. Nicolas, J.C., Ingrand, D. \& Fortier, B. A one year virological survey of acute intussusception in childhood. $J$ Med Virol 1982, 9: $267-271$.

5. Reijnen, H.A.M., Joosten, H.J.M. \& Boer, H.H.M. Diagnosis and treatment of adult intussusception. Am J Surg 1989, 158: 25-28.
6. Montali, G., Croce, F., De Pra, L. \& Sobiati, L. Intussusception of the bowel: a new sonographic pattern. Br J Radiol 1983, 56: $621-623$.

7. Alossi, V. \& Salerno, G. The 'Hay-Fork' sign in the ultrasonic diagnosis of intussusception. Gastrointest Radiol 1985, 10: $177-179$.

8. Kruse, J. \& Heath, R. Melanoma metastatic to the gastrointestinal tract. Am Family Physician 1990, 41: 165-168.

9. Agha, F.P. Intussusception in adults. Am J Radiol 1986, 146: 527-531. 